1 Nature Microbiology. Letter

2

\title{
3 Origins of pandemic Vibrio cholerae from environmental gene pools
}

4 B. Jesse Shapiro ${ }^{1}$, Inès Levade ${ }^{1 \#}$, Gabriela Kovacikova ${ }^{2 \#}$, Ronald K. Taylor ${ }^{2 \ddagger}$, Salvador

5 Almagro-Moreno ${ }^{2,3 *}$

6

$7 \quad{ }^{1}$ Department of Biological Sciences, University of Montreal, Montreal, Quebec, Canada.

$8{ }^{2}$ Department of Microbiology and Immunology, Geisel School of Medicine at Dartmouth,

9 Hanover, New Hampshire, USA. ${ }^{3}$ Burnett School of Biomedical Sciences, College of Medicine,

10 University of Central Florida, Orlando, Florida, USA.

11

12 *For correspondence: samoreno@ucf.edu

13 \#These authors contributed equally

$14 \quad{ }^{\ddagger}$ Deceased 


\section{Abstract}

16 Some microbes can transition from an environmental lifestyle to a pathogenic one ${ }^{1-3}$. This

17 ecological switch typically occurs through the acquisition of horizontally acquired virulence

18 genes ${ }^{4,5}$. However, the genomic features that must be present in a population prior to the

19 acquisition of virulence genes and emergence of pathogenic clones remain unknown. We

20 hypothesized that virulence adaptive polymorphisms (VAPs) circulate in environmental

21 populations and are required for this transition. We developed a comparative genomic

22 framework for identifying VAPs, using Vibrio cholerae as a model. We then characterized

23 several environmental VAP alleles to show that, while some of them reduced the ability of

24 clinical strains to colonize a mammalian host, other alleles conferred efficient host colonization.

25 These results show that VAPs are present in environmental bacterial populations prior to the

26 emergence of virulent clones. We propose a scenario in which VAPs circulate in the

27 environment, they become selected and enriched under certain ecological conditions, and finally

28 a genomic background containing several VAPs acquires virulence factors that allows for its

29 emergence as a pathogenic clone. 


\section{Main text}

31 Numerous bacterial pathogens have emerged from environmental populations ${ }^{1-3,6}$. These

32 virulent clones evolve through the acquisition of toxins and host colonization factors ${ }^{4,5}$. Given

33 that the genes encoding these factors can often spread widely by horizontal gene transfer, it is

34 surprising that only a limited number of pathogenic clones have emerged from any particular

35 bacterial species. As a model of how environmental gene pools give rise to pandemic clones,

36 we used Vibrio cholerae, a genetically diverse group of aquatic bacteria that include a confined

37 phylogenetic group, the "pandemic genome" group (PG), that can cause the severe diarrheal

38 disease cholera in humans ${ }^{7,8}$. Seven pandemics of cholera have been recorded to date, all

39 caused by the PG group. The current pandemic is caused by strains of the El Tor biotype, and

40 has spread across the globe in several waves of transmission ${ }^{9}$. Virulence in V. cholerae PG is

41 mainly determined by two virulence factors: the cholera toxin (CT) and the toxin-coregulated

42 pilus (TCP), which are encoded within horizontally acquired genetic elements, the CTXФ phage

43 and the Vibrio Pathogenicity Island-1 (VPI-1) respectively ${ }^{10-12}$. Both CTXФ and VPI-1 are always

44 found in the PG group, however, they are also encoded in some environmental populations of

45 V. cholerae ${ }^{13-15}$. Furthermore, even though some non-PG strains can cause gastrointestinal

46 infections, only strains from the PG clade have ever emerged as a source of pandemic

47 cholera $^{16}$.

48 To investigate the evolutionary origins of pandemic clones of $V$. cholerae and the

49 potential for their reemergence, we analyzed $43 \mathrm{~V}$. cholerae genomes sequenced from clinical

50 and environmental samples (Methods; Supplementary Table 1). These genomes span the

51 known genetic diversity of $V$. cholerae (Supplementary Notes), and were divided into a primary

52 dataset of 22 genomes and a replication dataset of 22 genomes, with one reference genome in

53 common (Supplementary Table 2). In the primary dataset, we chose 7 PGs, including both 
54 classical strains, the source of the first six pandemics, and El Tor, to represent the genetic

55 diversity of the pandemic group. We compared these with 15 non-clinical environmental

56 genomes (EGs): 10 EGs from worldwide samples to include global diversity, and five sympatric

57 isolates from the Great Bay Estuary (GBE) in New Hampshire, USA, a region with no recent

58 history of cholera outbreaks ${ }^{17}$.

Consistent with the results of previous studies ${ }^{7,9,17,18}$, PGs form a distinct monophyletic

60 group compared to EGs, based on the aligned core genome (Fig. 1a). Other than the PG group,

61 there is little phylogenetic structure and the tree is star-like in both datasets (Supplementary Fig.

62 1). Reticulations in the phylogenetic network indicate recombination or homoplasies (repeated

63 mutations in independent lineages at the same locus), consistent with a large, genetically

64 diverse and recombining $V$. cholerae population ${ }^{18,19}$ (Supplementary Fig. 1). Given such a

65 recombining population with mobile virulence factors, it remains puzzling why the ability to cause pandemic cholera is limited to the PG group.

To find unique features of PGs that could explain their pandemic success, we first searched for genes present in PGs but absent in EGs. Although there are a few genes and gene clusters which appear to be universally present in PGs, including CTXФ and VPI-1, none of these are unique to PGs as they are also present in some EGs (Supplementary Table 3).

71 This observation is consistent with previous work showing that these virulence genes are rapidly 72 gained and lost in both EGs and PGs ${ }^{7,18}$. Therefore, there appear to be no gene families whose

73 presence can easily explain the origin of pandemic cholera, nor are there strong boundaries to 74 gene transfer between PGs and EGs.

Given the lack of PG-specific genes, we hypothesized that the environmental ancestor of

76 the PGs had a particular genomic background containing alleles of core genes - which we term

77 virulence adaptive polymorphisms (VAPs) - that served as "preadaptations" and enhanced its

78 potential to give rise to pandemic disease. We started our search for VAPs by identifying SNPs 
79 in the aligned core of the 22 primary dataset genomes with one allele uniquely present in all

80 PGs and a different allele uniquely present in all EGs. We called this a "fixed" SNP pattern. We

81 identified 819 such fixed SNPs distributed across the genome (Fig. 1b; Supplementary Fig. 3).

82 Some fixed SNPs could have contributed to the evolution of the pandemic phenotype in PG,

83 while others could be selectively neutral hitchhikers on the PG genomic background. Using the

84 McDonald-Kreitman test (Methods), we found evidence for genome-wide positive selection

85 during the divergence of PGs from EGs, due to an excess of nonsynonymous changes at fixed

86 sites, in both datasets (Supplementary Table 4). However, no individual gene showed evidence

87 for selection after correcting for multiple tests (Methods), rendering it difficult to identify

88 candidate VAPs using fixed SNPs. Nonetheless, fixed SNPs constitute only a modest fraction of

89 possible SNP patterns (Supplementary Table 2), and VAPs could also exist at other SNP sites

90 that might shed light in the evolutionary past of the ancestral PG.

91 We then defined and searched for a "mixed" SNP pattern, where PGs encode one fixed

92 allele and EGs encode a "mix" of two alleles: one that is unique to EGs and also one that is

93 fixed in PGs (Fig. 1c inset). We reasoned that the existence of PG-like alleles segregating in

94 contemporary environmental populations of $V$. cholerae could be informative about: 1) pathogen

95 emergence, because having an allele fixed in PG suggests that it could also have been present

96 in the environmental ancestor of PG; and 2) the potential for pathogen reemergence, as PG-like

97 alleles, and thus potential VAPs, are still circulating in the environmental gene pool. We

98 identified 39,171 mixed SNPs in the primary dataset. Most genes contain few mixed SNPs

99 (median of 3) but some genes contain dense clusters, resulting in a mean of 10.3 mixed SNPs

100 per gene. The replication dataset contained greater genetic diversity, but showed the same

101 pattern of a few genes containing many mixed SNPs (Supplementary Table 2). Clusters of

102 genes containing many mixed SNPs are visible when plotted across the genome (Fig. 1c).

103 Some of these clusters are known polymorphic regions of the genome and could be mutation 
104 hotspots containing SNPs not directly relevant to virulence adaptation. However, clusters of

105 mixed SNPs do not visibly overlap with clusters of overall polymorphism (Fig. 1c), indicating that

106 accumulation of mixed SNPs cannot be explained only by mutation hotspots.

To formally exclude mutation hotspots and focus on clusters of mixed SNPs shaped mainly by natural selection instead of mutation and drift, we considered only genes with an excess of nonsynonymous (NS) mixed SNPs relative to synonymous (S) mixed SNPs, which

110 control for the baseline mutation rate. The mixed SNP pattern by definition groups PG-like EGs

111 away from the other environmental strains and clusters them with the PGs. We reasoned that

112 genes with an elevated NS:S ratio at mixed SNP sites were more likely to show phenotypic

113 variations and have evolved under positive selection, possibly underlying preadaptations of the

114 PG ancestor. Using a threshold of the number of mixed NS SNPs, NS:S, and $d N / d S$ all two

115 standard deviations above their genome-wide medians (Methods), we identified five genes as

116 candidate VAPs in the primary dataset, three of which survived multiple hypothesis correction,

117 and two of which (ompU and hypothetical gene VCD_001600) were also found in the replication

118 dataset (Table 1). In contrast to the star-like genome-wide phylogeny (Fig. 1a), each of these

119 five gene trees support one or more EGs grouping with PGs (Fig. 1d and Supplementary Fig. 4).

120 Three additional VAPs - all hypothetical proteins - were identified in the replication dataset,

121 suggesting the potential for other candidate VAPs to be identified with further sampling of

122 genetically diverse environmental genomes (Supplementary Table 5).

123 Among the candidate VAPs, the gene with the most significant excess of mixed

124 nonsynonymous SNPs in both datasets is ompU (Table 1). OmpU is an outer membrane porin

125 that has been shown to play numerous roles in the intestinal colonization of $V$. cholerae, making

126 it a compelling candidate for phenotypic characterization ${ }^{20-23}$. We observed that environmental

127 strains RC385, GBE0658and GBE0428 have PG-like ompU alleles whereas most

128 environmental strains, such as GBE1114, branch distantly from PG (Fig. 1d). We hypothesized 
129 that the PG-like alleles present in environmental strains might confer properties conducive to

130 virulence. To test this, we constructed three different mutant strains each encoding one of three

131 environmental alleles of ompU (all from sympatric GBE strains) into the background of N16961,

132 a clinical strain from the current pandemic (Fig. 2). OmpU was detected on a protein gel stained

133 with Coomassie blue and through immunoblot in all the constructed strains, indicating that all

134 the strains effectively produce the environmental versions of the protein (Fig. 2a and

135 Supplementary Fig. 6). The band size differences reflect the diversity in protein sizes among the

136 three environmental OmpU variants (Supplementary Fig. 5 and Supplementary Table 6).

137 We performed three sets of experiments to compare the phenotypes conferred by EG

138 and PG-like alleles of ompU. First, we determined the survival of these strains in the presence

139 of $0.4 \%$ bile, as it has been previously shown that OmpU confers resistance to this antimicrobial

140 compound ${ }^{20}$. The mutant strain encoding the ompU allele from the environmental strain

$141 \mathrm{GBE} 1114\left(\mathrm{OmpU} \mathrm{GBE1114}^{\mathrm{G}}\right)$ showed a similar survival rate in the presence of bile as a deletion

142 mutant (Fig 2b). In contrast, OmpU ${ }^{\mathrm{GBE} 0658}$ show similar survival in the presence of bile as a PG

143 strain (Fig. 2b). These experiments indicate that some environmental alleles of ompU confer

144 properties beneficial for virulence. Second, we tested the survival of the mutants in the presence

145 of polymyxin $\mathrm{B}$, as $\mathrm{OmpU}$ also confers resistance against this antibiotic ${ }^{21}$. The ability to tolerate

146 the antimicrobial effects of polymyxin B appears to be independent of which ompU allele is

147 encoded by $V$. cholerae, as the three strains encoding environmental alleles of ompU had a

148 similar survival rate (Fig. 2c). This experiment shows that OmpU GBE1114 is not simply a loss of

149 function mutant, and is not equivalent to a knockout. Third, we determined the intestinal

150 colonization of the ompU mutants by performing competition assays using the infant mouse

151 model of human infection. We found that OmpU ${ }^{\text {GBE1114 }}$ had a colonization defect similar to

$152 \Delta o m p U$ whereas $O \mathrm{mpU}^{\mathrm{GBE} 0658}$ was able to colonize similarly to the strain with the wild-type PG 
153 allele (Fig. 2d). These results indicate that certain naturally occurring environmental alleles of

154 ompU confer properties that provide an advantage to $V$. cholerae during or prior to host

155 colonization, as would be expected for VAPs.

156 The ompU $U^{G B E 1114}$ allele appears to be maladaptive for intestinal colonization; however, its

157 presence in several environmental isolates of $V$. cholerae prompted us to investigate its

158 possible adaptive role in the environment (Fig. 2e). V. cholerae forms biofilms on the surface of

159 biotic and abiotic environmental surfaces ${ }^{24-26}$, yet biofilm formation inside the host is thought to

160 impair intestinal colonization ${ }^{22,27}$. Strains with deletions in ompU have been shown to form a

161 more robust biofilm on abiotic surfaces ${ }^{25}$. We found that OmpU ${ }^{\text {GBE1114 }}$ has higher biofilm

162 formation than the strain encoding the wild-type PG allele, similar to the $\Delta$ ompU strain (Fig. 2e).

163 Both OmpU ${ }^{\mathrm{GBE} 0658}$ and $\mathrm{OmpU} \mathrm{GBE}^{\mathrm{GB} 28}$ formed biofilm similar to the strains with the wild-type PG

164 allele (Fig. 2e). It therefore appears that there is an evolutionary trade-off between encoding the

165 PG-like or EG-like alleles of VAPs, as they seem to confer mutually exclusive traits: either

166 biofilm formation or bile resistance and host colonization. This suggests that environmental

167 strains can be divided into subgroups which, due to their contrasting lifestyles, differ in their

168 potential to give rise to pandemic clones.

We have determined that virulence adaptive polymorphisms are present in the

170 environment, and shown how these VAPs can be identified, based on two independent sets of

171 genomes. The top candidate VAP, ompU, was identified in both of our genomic datasets. Our

172 experiments show that the ompU allele from some environmental strains, such as GBE0658,

173 confers properties that allow for host colonization equally as efficient as alleles from clinical

174 strains (Fig. 2). This leads to a natural question: Why have environmental strains with PG-like

175 alleles not emerged as pandemic cholera strains? It appears that a variety of virulence adaptive 
176 genes and alleles are circulating in the environment, but only the PG group encodes the optimal

177 combination of VAPs that allowed for pandemic potential (Fig. 3). We propose a conceptual

178 model in which VAPs circulate in a diverse, recombining environmental gene pool, being

179 maintained in the population through various biotic and abiotic selective pressures (Fig. 3a). A

180 new ecological opportunity occurs, such as human consumption of brackish water or transient

181 colonization of other animal hosts, which leads to the proliferation and gradual enrichment in the

182 population of clones encoding a mosaic of VAPs (Fig. 3b). Finally, a genome encoding a critical

183 combination of VAPs acquires key virulence factors allowing it to emerge as a virulent,

184 potentially pandemic clone (Fig. 3c).

185 Our model posits that VAPs are circulating in the environment prior to the acquisition of

186 key virulence factors. This is based on experimental evidence that a current pandemic strain

187 cannot efficiently colonize the mammalian intestine without a PG-like ompU allele. If virulence

188 adaptive alleles of $o m p U$ are indeed required prior to the acquisition of virulence factors, we

189 would expect the same phenotypes of PG-like and EG-like ompU alleles in the genomic

190 background of a more deeply branching PG isolate, such as classical V. cholerae. Indeed, we

191 found that PG-like ompU alleles in the classical O395 background conferred efficient host

192 colonization (Supplementary Fig. 7), which is consistent with an ompU VAP having played a role

193 in the emergence of pandemic V. cholerae.

194 Our model further postulates that virulence adaptive alleles become enriched in the

195 environmental population. Such enrichment would be made possible if these alleles provided a

196 selective advantage in a newly available ecological niche, such as a human population

197 consuming brackish water ${ }^{28}$. In previous work, we modeled an evolving, recombining microbial

198 population that encounters a new ecological opportunity ${ }^{29}$. When adaptation to the new niche

199 depends on few loci under positive selection in the niche, it is more likely for recombination to

200 assemble the right combination of alleles in the same genome. These loci (akin to VAPs) could 
201 contribute additively or synergistically to fitness. For example, the PG-like ompU alleles confer a

202 tenfold increase in fitness during host colonization (Fig. 2d). Other VAPs might contribute further

203 to this enhancement in host colonization. As more loci are involved in adaptation, it becomes

204 less likely to achieve the optimal combination. Assuming the $V$. cholerae population undergoes

205 approximately 100 recombination events per locus per generation (6.5 recombination events for

206 every point mutation $)^{19}$, equivalent to a recombination rate of $10^{-4}$ in the modelled population of

207 size $10^{6}$, an optimal combination of alleles at five loci could conceivably evolve, but seven loci is

208 very unlikely ${ }^{29}$. Therefore, if virulence depended on five or fewer positively selected loci in the $V$.

209 cholerae genome, the optimal combination of alleles would be expected to appear repeatedly in

210 nature. Given suitable ecological opportunities, it is then plausible that pandemic V. cholerae

211 could emerge multiple times, originating from outside the PG group. However, the number of

212 loci that are sufficient for the emergence of a virulent strain remains unknown and if it was much

213 greater than five, pathogen emergence would be naturally limited. We identified eight candidate

214 VAPs in our two datasets, and if all eight are phenotypically confirmed to be VAPs, this number

215 of VAPs $(>5)$ might naturally limit pandemic clone emergence. Furthermore, these eight

216 candidate VAPs passed stringent filters and we suspect there might exist additional VAPs in the

217 genome, identifiable by further sampling and experimentation.

Here we have described a framework for identifying loci that are present in a natural

219 population and confer properties beneficial for virulence prior to acquisition of essential

220 virulence genes and host colonization. This framework could be applied to other bacterial

221 pathogens that emerge as clonal offshoots from non-virulent relatives, including Yersinia,

222 Salmonella, Escherichia, or other pathogenic Vibrio species ${ }^{1-4,6}$. Pathogens that emerged

223 through clonal expansion limit our ability to dissect the genetic basis of their pathogenicity,

224 because bacterial genome-wide association studies lack power when the phenotype of interest

225 has evolved only once ${ }^{30}$. Our framework therefore provides a way forward to identify the genetic 
bioRxiv preprint doi: https://doi.org/10.1101/063115; this version posted December 13, 2016. The copyright holder for this preprint (which was not certified by peer review) is the author/funder, who has granted bioRxiv a license to display the preprint in perpetuity. It is made available under aCC-BY-NC-ND 4.0 International license.

226 basis of virulence, even in pathogens that evolved through clonal expansion, and begin to 227 assess the risk of pathogen emergence and reemergence from environmental gene pools. 


\section{Methods}

229 Genome sequencing. DNA from clinical isolates (Bgd1, Bgd5, Bgd8, MQ1795 ${ }^{31,32}$ ) and

230 environmental isolates (GBE0428, GBE0658, GBE1068, GBE1114, GBE1173 ${ }^{17}$ ) was extracted

231 using the Gentra kit (QIAGEN) and purified using the MoBio PowerClean Pro DNA Clean-Up

232 Kit. Multiplexed genomic libraries were constructed using the Illumina-compatible Nextera DNA

233 Sample Prep kit following the manufacturer's instructions. Sequencing was performed with 250-

234 bp paired-end (v2 kit) reads on the illumina MiSeq.

236 Genome assembly. To exclude low-quality data, raw reads were filtered with Trimmomatic ${ }^{33}$.

237 The 15 first bases of each reads were trimmed and reads containing at least one base with a

238 quality score of $<30$ were removed. De novo assembly was performed on the resulting reads

239 using Ray v2.3.1 ${ }^{34}$

241 Genome alignment, annotation and SNP calling. We used mugsy v. 1 r.2.2. $2^{35}$ with default

242 parameters to align the primary dataset of $22 \mathrm{~V}$. cholerae genomes (Supplementary Table 1).

243 From this alignment, we extracted dimorphic SNP sites and annotated genes according to MJ-

2441236 as a reference genome. We defined the core genome as locally colinear blocks (LCBs)

245 with all 22 genomes present in the alignment. We replicated the alignment, annotation and SNP

246 calling using 21 different $V$. cholerae genomes, mainly from Orata et al. ${ }^{18}$, plus the MJ-126

247 reference (Supplementary Table 1).

249 Definition of orthologous groups. Genomes were annotated using the RAST web server

250 (www.rast.nmpdr.org) ${ }^{36}$. Annotated genes were clustered into orthologous groups using

251 OrthoMCL (www.orthomcl.org) ${ }^{37}$ with default parameters, yielding 2844 orthologous groups. 
253 Phylogenetic analysis. We constructed a core genome phylogeny using the concatenated 254 alignment of 1031 single-copy orthologous protein-coding genes (present in exactly one copy in 255 each of the 22 primary dataset genomes). Each protein sequence was aligned with Muscle ${ }^{38}$, 256 and the concatenated alignment was used to infer an approximate maximum likelihood 257 phylogeny with FastTree v. $2.1 .8^{39}$ using default parameters (Fig. 1a). Individual gene trees (Fig.

258 1d) were built in the same way. We constructed a neighbour-net of the 22 genomes using

259 SplitsTree v.4.10 ${ }^{40}$, based on dimorphic SNPs from the mugsy genome alignment, excluding 260 sites with gaps.

262 Tests for selection. We conducted a genome-wide version of the McDonald-Kreitman test ${ }^{41}$ by

263 first counting the number of fixed nonsynonymous (fn), fixed synonymous (fs), polymorphic

264 nonsynonymous (pn), and polymorphic synonymous (ps) sites within each gene. We then

265 summed these values across all genes (FN, FS, PN, and PS) and calculated the genome-wide

266 Fixation Index, FI=(FN/FS)/(PN/PS). A fixation index greater than one suggests positive

267 selection between the ingroup and outgroup (in this case, between EGs and PGs). However,

268 care must be taken when computing a genome-wide FI because summing genes with different 269 amounts of substitutions and polymorphism can result in FI>1 in the absence of selection ${ }^{42}$. We

270 therefore performed 1000 permutations of the data, keeping the row totals $(f n+f s$ and $p n+p s$ )

271 and column totals (fn+pn and fs+ps) constant and recomputing FI. We used the mean FI from

272 the permutations as the expected value of FI under neutral evolution. To evaluate the

273 hypothesis that the observed FI was higher than expected, suggesting positive selection, we

274 computed a P-value as the fraction of permutations with $\mathrm{FI}$ greater or equal to the observed FI. 
275 We repeated the test using polymorphism from either the PG group or the EG group

276 (Supplementary Table 3).

277 To identify individual genes under selection between PGs and EGs in the primary

278 dataset, we restricted our search to 87 genes with $\mathrm{fn}>0.681$ and fn:fs $>1.48$ (respectively two

279 standard deviations about the genome-wide medians). We then calculated the gene-specific FI

280 and assessed its significance with a Fisher exact test. We found no genes with FI significantly

281 greater than one, after Bonferroni correction for 87 tests. Similarly, in the replication dataset, we

282 restricted our search to 26 genes with $\mathrm{fn}>1.5$ and fn:fs $>1.70$, none of which had significantly

283 high FI after correction for multiple tests.

284 To identify genes with an excess of nonsynonymous mixed SNPs (likely due to selection

285 for amino acid changes), we restricted our search to five genes with $\geq 12$ NS mixed SNPs per

286 gene and mixed NS:S > 1.78 (respectively two standard deviations above the genome-wide

287 medians). We used a one-sided binomial test to assess whether the observed NS:S ratio for

288 each gene was significantly greater than the genome-wide median NS:S ratio of 0.5 (after

289 adding a pseudocount of one to both NS and S). Three out of the five genes had a significantly

290 high mixed NS:S ratio $(P<0.05)$ after Bonferroni correction for five tests (Table 1). We repeated

291 this procedure in the replication dataset, identifying genes with $\geq 18$ NS mixed SNPs per gene

292 and mixed NS:S > 1.65 (respectively two standard deviations above the genome-wide

293 medians). We used a one-sided binomial test to assess whether the observed NS:S ratio for 
294 each gene was significantly greater than the genome-wide median NS:S ratio of 0.33 (after

295 adding a pseudocount of one to both NS and S). The results of these tests are shown for genes

296 also identified in the primary dataset (Table 1) and three additional genes identified in the

297 replication dataset (Supplementary Table 5).

Bacterial strains and plasmids. V. cholerae O395 and V. cholerae N16961 were used as wild-

300 type strains of classical and El Tor biotypes respectively. Strains cultivated on solid medium

301 were grown on LB agar; strains in liquid media were grown in aerated LB broth at $37^{\circ} \mathrm{C}$.

302 pKAS154 was used for allelic exchange ${ }^{43}$. When necessary, media was supplemented with

303 antibiotics to select for certain plasmids or strains of $V$. cholerae at the following concentrations:

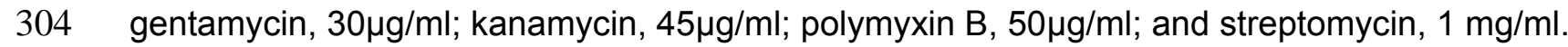

306 Strain construction. In-frame deletions and exchange of ompU alleles in both $\mathrm{O} 395$ and

307 N16961 biotypes were constructed via homologous recombination ${ }^{43}$. For ompU deletions, PCR

308 was used to amplify two $500 \mathrm{bp}$ fragments flanking the ompU gene and to introduce restriction

309 sites for cloning into the suicide vector pKAS154. For exchange of environmental ompU alleles,

310 the respective allele was also amplified using the extracted DNA from each environmental

311 strain. Restriction sites were introduced in the primers. The fragments were then cloned into a

312 restriction-digested suicide plasmid, pKAS154, using a four-segment ligation for each

313 environmental allele exchange mutants (plasmid, ompU flanking fragments and environmental

314 ompU allele). The resulting plasmids were electroporated into Escherichia coli S17-1גpir. E. coli

315 with the constructed inserts. Different sizes of ompU genes were confirmed, sequenced and 
compared to the genome sequences of environmental strains. For both deletion and exchange

317 mutants, plasmids with the insert of interest were mated with wild-type $V$. cholerae 0395 or

318 N16961, and allelic exchange was carried out by selection on antibiotics as described

319 previously ${ }^{43}$. For a more detailed description of allelic exchange please refer to Skorupski and

320 Taylor $1998^{43}$. Potential mutants were screened using PCR: two primers flanking the deletion

321 construct were used to amplify chromosomal DNA isolated from plated $V$. cholerae. The lengths

322 of the PCR fragments were analyzed on $0.8 \%$ agarose gel for gene deletions and putative

323 deletions and allele exchange were subsequently confirmed by DNA sequencing.

325 OmpU visualization and immunobloting. Whole cell protein extracts were prepared from

326 cultures grown for overnight at $37^{\circ} \mathrm{C}$ in a rotary shaker. The extracts were subjected to SDS-

327 PAGE on 16\% Tris Glycine gels (Invitrogen). OmpU bands were visualized after protein gels

328 were stained by Coomassie blue overnight. Prior to staining the gels were transferred to

329 nitrocellulose membranes using iBlot (Invitrogen). The membranes were blocked O/N in Tris-

330 Buffered Saline, 3\% BSA. Primary OmpU antibodies were diluted 1:10,000 in TBST (Tris-

331 Buffered Saline, 0.5\% Tween-20). Membranes were incubated with primary antibodies for 2

332 hours at room temperature. After incubation, the membranes were washed with TBST four

333 times. Goat anti-rabbit secondary antibodies (BioRad) were diluted 1:10,000 in TBST and

334 incubated for 30 minutes at room temperature. The membranes were washed 4 times with TBS

335 (Tris-Buffered Saline). Reactive protein bands were detected via ECL (Amersham).

337 Survival assays. V. cholerae strains were cultured overnight in LB broth at $37^{\circ} \mathrm{C}$ in a rotary

338 shaker. Overnight cultures were diluted 1:100 in LB and grown to an OD600 of 0.5. Cells were 
339 pelleted and resuspended in either LB broth, LB containing $0.4 \%$ bile bovine (Sigma), or LB

340 containing $1000 \mathrm{U} / \mathrm{ml}$ of polymyxin $\mathrm{B}$ (Sigma). Cultures were incubated for $1 \mathrm{~h}$ at $37^{\circ} \mathrm{C}$ in a rotary

341 shaker. After incubation CFU/ml of each culture was calculated by plating dilutions in LB plates.

342 Survival was calculated by comparing the number of CFU/ml in LB plus treatment versus LB.

$343 \quad \mathrm{~N} \geq 6$. No samples were excluded.

345 Infant mouse competition assays. Overnight cultures were diluted 1:100. Each test strain was

346 mixed in a 1:1 ratio with a $\Delta$ lacZ reference strain. Four- to five-day-old CD-1 mice (Mus

347 musculus) from several mixed litters were randomly inoculated orogastrically in blinded

348 experiments with $50 \mu$ l of the bacterial mixture. Sex of the animals was not inspected prior to

349 inoculations. The intestines were harvested $24 \mathrm{~h}$ post-inoculation and homogenized in $4 \mathrm{ml}$ of LB

350 broth containing 10\% glycerol. The mixtures were serially diluted and plated on LB agar plates

351 supplemented with streptomycin and 5-bromo-4-chloro-3-indolyl-D-galactopyramoside (X-Gal)

$352(40 \mu \mathrm{g} / \mathrm{ml})$. The competition indices were calculated as previously described by others, test

353 (output CFUs/input CFUs)/reference (output CFUs/ input CFUs) and the sample size selected

354 was appropriate for statistical analysis. No samples or animals were excluded. Animal work was

355 approved by the Institutional Animal Care and Use Committee (IACUC).

357 Biofilm assays. 96-well plate assay. Cultures were incubated overnight at $30^{\circ} \mathrm{C} .100 \mu \mathrm{l}$ of $1: 100$

358 dilutions of overnight cultures were placed per well in 96 -well plates. Plates were left at $25^{\circ} \mathrm{C}$ for

359 24h. Liquid contents were discarded and plates were washed 2 times with LB. $200 \mu$ of $0.01 \%$ 
360 crystal violet was added per well and incubated at room temperature for 5 minutes. Liquid

361 contents were discarded and plates were washed extensively with $\mathrm{dH}_{2} \mathrm{O}$. After the plates were

362 dry, biofilms were resuspended in $150 \mu$ of $50 \%$ acetic acid. Contents were transferred to a flat

363 bottom dish and quantitated in a microtiter plate reader at OD550. Values were plotted using

364 Prism software. $\mathrm{N}=15$. 


\section{Data availability}

366 The nine genomes sequenced in this study (Bgd1, Bgd5, Bgd8, MQ1795, GBE0428, GBE0658,

367 GBE1068, GBE1114, GBE1173) have been deposited under BioProject ID PRJNA349157 in

368 NCBI GenBank under accession numbers SAMN05924900-SAMN05924908 (Supplementary

369 Table 1). All other data supporting the findings of this study are available from the authors upon

370 request. 


\section{References}

373 1. McNally, A., Thomson, N. R., Reuter, S. \& Wren, B. W. 'Add, stir and reduce': Yersinia spp. as model

bacteria for pathogen evolution. Nat. Rev. Microbiol. 14, 177-190 (2016).

2. Luo, C. et al. Genome sequencing of environmental Escherichia coli expands understanding of the ecology and speciation of the model bacterial species. Proc. Natl. Acad. Sci. U.S.A. 108, 7200-7205 (2011).

3. Reen, F. J., Almagro-Moreno, S., Ussery, D. \& Boyd, E. F. The genomic code: inferring Vibrionaceae niche specialization. Nat. Rev. Microbiol. 4, 697-704 (2006).

4. Ochman, H. Genes Lost and Genes Found: Evolution of Bacterial Pathogenesis and Symbiosis. Science 292, 1096-1099 (2001).

5. Shapiro, B. J. How clonal are bacteria over time? Curr. Opin. Microbiol. 31, 116-123 (2016).

6. Cui, Y. et al. Epidemic Clones, Oceanic Gene Pools, and Eco-LD in the Free Living Marine Pathogen Vibrio parahaemolyticus. Mol. Biol. Evol. 32, 1396-1410 (2015).

7. Chun, J. et al. Comparative genomics reveals mechanism for short-term and long-term clonal transitions in pandemic Vibrio cholerae. Proc. Natl. Acad. Sci. U.S.A. 106, 15442-15447 (2009).

8. Boucher, Y. Sustained local diversity of Vibrio cholerae $\mathrm{O} 1$ biotypes in a previously cholera-Free country. mBio 7, e00570-16 (2016).

9. Mutreja, A. et al. Evidence for several waves of global transmission in the seventh cholera pandemic. Nature 477, 462-465 (2011)

10. Taylor, R. K., Miller, V. L., Furlong, D. B. \& Mekalanos, J. J. Use of phoA gene fusions to identify a pilus colonization factor coordinately regulated with cholera toxin. Proc. Natl. Acad. Sci. U.S.A. 84, 2833-2837 (1987).

11. Waldor, M. K. \& Mekalanos, J. J. Lysogenic conversion by a filamentous phage encoding cholera toxin. Science (New York, N.Y.) 272, 1910-1914 (1996).

12. Karaolis, D. K. et al. A Vibrio cholerae pathogenicity island associated with epidemic and pandemic strains. Proc. Natl. Acad. Sci. U.S.A. 95, 3134-3139 (1998).

13. Faruque, S. M. et al. Genetic diversity and virulence potential of environmental Vibrio cholerae population in a cholera-endemic area. Proc. Natl. Acad. Sci. U.S.A. 101, 2123-2128 (2004).

14. Rivera, I. N., Chun, J., Huq, A., Sack, R. B. \& Colwell, R. R. Genotypes associated with virulence in environmental isolates of Vibrio cholerae. Appl. Environ. Microbiol. 67, 2421-2429 (2001).

15. Gennari, M., Ghidini, V. \& Lleo, M. M. Virulence genes and pathogenicity islands in environmental Vibrio strains non-pathogenic to humans. FEMS Microbiol. Ecol. 82, 563-573 (2012).

16. Dziejman, M. et al. Genomic characterization of non-O1, non-O139 Vibrio cholerae reveals genes for a type III secretion system. Proc. Natl. Acad. Sci. U.S.A. 102, 3465-3470 (2005).

17. Schuster, B. M. et al. Ecology and genetic structure of a northern temperate Vibrio cholerae population related to toxigenic isolates. Appl. Environ. Microbiol. 77, 7568-7575 (2011).

18. Orata, F. D. et al. The dynamics of genetic interactions between Vibrio metoecus and Vibrio cholerae, two close relatives co-occurring in the environment. Genome Biol Evol 7, 2941-2954 (2015).

19. Keymer, D. P. \& Boehm, A. B. Recombination shapes the structure of an environmental Vibrio cholerae population. Appl. Environ. Microbiol. 77, 537-544 (2011).

20. Provenzano, D., Schuhmacher, D. A., Barker, J. L. \& Klose, K. E. The virulence regulatory protein ToxR mediates enhanced bile resistance in Vibrio cholerae and other pathogenic Vibrio species. Infect. Immun. 68, 1491-1497 (2000).

21. Mathur, J. \& Waldor, M. K. The Vibrio cholerae ToxR-regulated porin OmpU confers resistance to antimicrobial peptides. Infect. Immun. 72, 3577-3583 (2004).

22. Almagro-Moreno, S., Pruss, K. \& Taylor, R. K. Intestinal colonization dynamics of Vibrio cholerae. PLoS Pathog. 11, e1004787 (2015).

23. Merrell, D. S., Bailey, C., Kaper, J. B. \& Camilli, A. The ToxR-mediated organic acid tolerance response of Vibrio cholerae requires OmpU. J. Bacteriol. 183, 2746-2754 (2001).

24. Watnick, P. I. \& Kolter, R. Steps in the development of a Vibrio cholerae El Tor biofilm. Mol. Microbiol. 34, 586-595 (1999).

25. Valeru, S. P., Wai, S. N., Saeed, A., Sandström, G. \& Abd, H. ToxR of Vibrio cholerae affects biofilm, rugosity and survival with Acanthamoeba castellanii. BMC Res Notes 5, 33 (2012).

26. Yildiz, F. H. \& Schoolnik, G. K. Vibrio cholerae O1 El Tor: identification of a gene cluster required for the rugose colony type, exopolysaccharide production, chlorine resistance, and biofilm formation. Proc. Natl. Acad. Sci. U.S.A. 96, 4028-4033 (1999). 
27. Hsiao, A., Liu, Z., Joelsson, A. \& Zhu, J. Vibrio cholerae virulence regulator-coordinated evasion of host immunity. Proc. Natl. Acad. Sci. U.S.A. 103, 14542-14547 (2006).

28. Boucher, Y., Orata, F. D. \& Alam, M. The out-of-the-delta hypothesis: dense human populations in low-lying river deltas served as agents for the evolution of a deadly pathogen. Front. Microbiol. 6, L19401 (2015).

29. Friedman, J., Alm, E. J. \& Shapiro, B. J. Sympatric Speciation: When Is It Possible in Bacteria? PLoS ONE 8, e53539 (2013).

30. Chen, P. E. \& Shapiro, B. J. The advent of genome-wide association studies for bacteria. Curr. Opin. Microbiol. 25, 17-24 (2015).

31. Nair, G. B. et al. New variants of Vibrio cholerae 01 biotype El Tor with attributes of the classical biotype from hospitalized patients with acute diarrhea in Bangladesh. J. Clin. Microbiol. 40, 3296-3299 (2002).

32. Son, M. S., Megli, C. J., Kovacikova, G., Qadri, F. \& Taylor, R. K. Characterization of Vibrio cholerae O1 El Tor biotype variant clinical isolates from Bangladesh and Haiti, including a molecular genetic analysis of virulence genes. J. Clin. Microbiol. 49, 3739-3749 (2011).

33. Bolger, A. M., Lohse, M. \& Usadel, B. Trimmomatic: a flexible trimmer for Illumina sequence data. Bioinformatics 30, 2114-2120 (2014).

34. Boisvert, S., Raymond, F., Godzaridis, É., Laviolette, F. \& Corbeil, J. Ray Meta: scalable de novo metagenome assembly and profiling. Genome Biol 13, R122 (2012).

35. Samuel V Angiuoli, S. L. S. Mugsy: fast multiple alignment of closely related whole genomes. Bioinformatics 27, 334-342 (2011).

36. Aziz, R. K. et al. The RAST Server: Rapid Annotations using Subsystems Technology. BMC Genomics $\mathbf{9 ,} 1$ (2008).

37. Li, L., Stoeckert, C. J. \& Roos, D. S. OrthoMCL: identification of ortholog groups for eukaryotic genomes. Genome Res. 13, 2178-2189 (2003).

38. Edgar, R. C. MUSCLE: multiple sequence alignment with high accuracy and high throughput. Nucleic Acids Res. 32, 1792-1797 (2004).

39. Price, M. N., Dehal, P. S. \& Arkin, A. P. FastTree 2 - Approximately Maximum-Likelihood Trees for Large Alignments. PLoS ONE 5, e9490 (2010).

40. Bryant, D. \& Moulton, V. Neighbor-net: an agglomerative method for the construction of phylogenetic networks. Mol. Biol. Evol. 21, 255-265 (2004).

41. McDonald, J. H. \& Kreitman, M. Adaptive protein evolution at the Adh locus in Drosophila. Nature 351, 652654 (1991).

42. Shapiro, J. A. et al. Adaptive genic evolution in the Drosophila genomes. Proc. Natl. Acad. Sci. U.S.A. 104, 2271-2276 (2007).

43. Skorupski, K. \& Taylor, R. K. Positive selection vectors for allelic exchange. Gene 169, 47-52 (1996). 
bioRxiv preprint doi: https://doi.org/10.1101/063115; this version posted December 13, 2016. The copyright holder for this preprint (which was not certified by peer review) is the author/funder, who has granted bioRxiv a license to display the preprint in perpetuity. It is made available under aCC-BY-NC-ND 4.0 International license.

462 Correspondence should be addressed to:

463 Salvador Almagro-Moreno (samoreno@ucf.edu) 


\section{Acknowledgements}

465 The authors would like to thank the anonymous reviewers for their thoughtful comments and

466 suggestions. We would also like to thank Otto Cordero, Yves Terrat, Nicolas Tromas and

467 Britney Privett for constructive comments on the manuscript. We thank Lawrence Shelven for

468 his highly valuable technical assistance. BJS was supported by a Canada Research Chair and

469 the Canadian Institutes for Health Research. RKT was supported by a National Institutes of

470 Health grants AI039654 and AI025096. SAM was supported by startup funds from the Burnett

471 School of Biomedical Sciences at the University of Central Florida and Dartmouth College's E.

472 E. Just Postdoctoral Fellowship. This article is dedicated to the memory of Ronald K. Taylor. 


\section{Author contributions}

474 SAM conceived the study. BJS, RKT and SAM designed the study. IL sequenced genomes.

475 BJS performed computational analysis. GK and SAM performed phenotypic characterization.

476 BJS and SAM analyzed, interpreted data and wrote the article. All authors have read a

477 version of the manuscript. 


\section{$478 \quad$ Figure Legends}

479 Figure 1. Comparative genomics reveals candidate virulence adaptive polymorphisms. a,

480 Phylogeny of $22 \mathrm{~V}$. cholerae genomes based on 1031 single-copy orthologs in the primary

481 dataset. All branches have local support values $>0.99$ (based on FastTree's approximate

482 likelihood ratio test) except for very short, deep internal branches (resulting in the star-like

483 polytomy at the centre of the tree). Not all 22 genomes are visible because some have nearly

484 identical sequences (e.g. 6 of the 7 PG genomes are nearly identical, shown as an orange

485 triangle; GBE1173 and GBE1114 are nearly identical, as can be seen in Supplementary Fig. 1).

486 b, Distribution of fixed SNPs across chromosome 1. (See Supplementary Fig. 3 for

487 chromosome 2). Genome position is according to the MJ-1236 reference genome. SNP-free

488 regions (e.g. near $3 \mathrm{Mbp}$, the locus of the integrative conjugative element) are part of the flexible

489 genome, present in the reference but not the other 21 genomes. The schematic tree in the top

490 left illustrates the fixed SNP pattern, in which one allele is present in PGs and a different allele

491 in EGs. c, Distribution of mixed SNPs across the genome. The cartoon tree in the top left

492 illustrates the mixed SNP pattern, in which one allele is fixed in PGs, and another allele is

493 polymorphic among EGs, with some EGs containing the PG-like allele. Black arrows show

494 candidate VAPs (Table 1). Grey arrow shows the flagellum as an example variable region not

495 containing candidate VAPs. d, ompU phylogeny. All visible branches have local support values

$496>0.9$ except for the branch separating RC385 and GBE0658, the branch grouping MJ-1236 and

497 O395 together, and the branch grouping HE09 and VL426 together.

499 Figure 2. Phenotypic characterization of ompU alleles. a, OmpU production in clinical strains

500 of $V$. cholerae encoding environmental alleles of ompU. Total protein lysates were run on a $16 \%$

501 Tris-glycine gel. OmpU bands were visualized after protein gels were stained with Coomassie 
502 blue. Three independent sets of protein lysates were examined and showed an identical band

503 pattern. $\mathbf{b}$, Survival of ompU mutants in the presence of bile $(n=7)$ or $\mathbf{c}$, polymyxin $B(n=6)$. $\mathbf{d}$,

504 Colonization of the small intestine of ompU mutant strains $(\mathrm{n}=6)$. e, Biofilm formation of ompU

505 mutant strains on an abiotic surface $(n=15)$. Yellow bars and symbols, PG-like allele; red bars

506 and squares, $\Delta o m p U$; blue bars and triangles, EG-like allele. Center values represent the mean

507 and error bars the standard deviation. Variance between the groups was similar. Statistical

508 comparisons were made using Student's $t$-test. ${ }^{*} P<0.05,{ }^{* *} P<0.001$

Figure 3. Model of pandemic clone emergence from an environmental gene pool. We

511 propose a model that involves three events required for the emergence of pathogenic clones

512 from environmental populations. a, selection of VAPs. Virulence adaptive alleles circulate in

513 naturally occurring populations (orange symbols) and can be exchanged and mobilized through

514 recombination (green dashed arrows). Ecological variation (e.g. temperature, nutrient

515 availability, $\mathrm{pH}$, etc.) leads to the selection of VAPs and an increase in their distribution in

516 environmental populations. b, enrichment of clones. A new ecological opportunity occurs

517 (human consumption of untreated waters, transient colonization of new environmental hosts,

518 etc.) which leads to the proliferation and enrichment in the population of clones encoding a

519 mosaic of VAPs. c, acquisition of virulence factors. A strain encoding a minimum set of VAPs

520 required for host colonization acquires the virulence factors that are necessary to produce a

521 successful infection and give rise to pandemic disease. 


\section{Tables}

524 Table 1. Characteristics of five predicted VAPs with an excess of nonsynonymous mixed 525 SNPs.

\begin{tabular}{|c|c|c|c|c|c|c|c|c|c|c|}
\hline & $\begin{array}{l}\text { Gene ID } \\
\text { (VCD \#) }\end{array}$ & Annotation & $\begin{array}{l}\text { Gene } \\
\text { length } \\
\text { (bp) }\end{array}$ & $\begin{array}{l}\text { Total } \\
\# \\
\text { SNPs }\end{array}$ & $\begin{array}{l}\text { Mixed } \\
d N\end{array}$ & $\begin{array}{l}\text { Mixed } \\
d S\end{array}$ & $\begin{array}{l}\text { Mixed } \\
d N / d S\end{array}$ & $\begin{array}{l}\text { Mixed } \\
\text { NS }\end{array}$ & $\begin{array}{l}\text { Mixed } \\
\mathbf{S}\end{array}$ & $P$ \\
\hline 1 & 003778 & $\begin{array}{l}\text { outer } \\
\text { membrane } \\
\text { protein OmpU }\end{array}$ & 1053 & $\begin{array}{l}204 \\
(110)\end{array}$ & $\begin{array}{l}0.037 \\
(0.018)\end{array}$ & $\begin{array}{l}0.045 \\
(0.033)\end{array}$ & $\begin{array}{l}0.83 \\
(0.53)\end{array}$ & $\begin{array}{l}28 \\
(13)\end{array}$ & $\begin{array}{l}11 \\
(8)\end{array}$ & $\begin{array}{l}0.0047^{*} \\
\left(0.0068^{*}\right)\end{array}$ \\
\hline 2 & 001600 & hypothetical & 258 & $\begin{array}{l}21 \\
(26)\end{array}$ & $\begin{array}{l}0.082 \\
(0.12)\end{array}$ & $\left|\begin{array}{l}0.081 \\
(0.065)\end{array}\right|$ & $\begin{array}{l}1.01 \\
(1.82)\end{array}$ & $\begin{array}{l}15 \\
(22)\end{array}$ & $\begin{array}{l}4 \\
(3)\end{array}$ & $\begin{array}{l}0.0096^{*} \\
\left(2.32 \mathrm{e}-8^{*}\right)\end{array}$ \\
\hline 3 & 001013 & hypothetical & 642 & $\begin{array}{l}19 \\
(13)\end{array}$ & $\begin{array}{l}0.034 \\
(0.006)\end{array}$ & $\left|\begin{array}{l}0.029 \\
(0.012)\end{array}\right|$ & $\begin{array}{l}1.18 \\
(0.55)\end{array}$ & $\begin{array}{l}15 \\
(2)\end{array}$ & $\begin{array}{l}4 \\
(1)\end{array}$ & $\begin{array}{l}0.0096^{*} \\
\text { (n.s.) }\end{array}$ \\
\hline 4 & 001209 & $\begin{array}{l}\text { MSHA } \\
\text { biogenesis } \\
\text { protein MshP }\end{array}$ & 432 & $\begin{array}{l}85 \\
(63)\end{array}$ & $\begin{array}{l}0.047 \\
(0.025)\end{array}$ & $\left|\begin{array}{l}0.045 \\
(0.054)\end{array}\right|$ & $\begin{array}{l}1.04 \\
(0.46)\end{array}$ & $\begin{array}{l}14 \\
(7)\end{array}$ & $\begin{array}{l}4 \\
(5)\end{array}$ & $\begin{array}{l}0.0154 \\
(0.066)\end{array}$ \\
\hline 5 & 001230 & $\begin{array}{l}\text { lipid A core O- } \\
\text { antigen ligase }\end{array}$ & 1794 & $\begin{array}{l}75 \\
(47)\end{array}$ & $\begin{array}{l}0.0098 \\
(0.001)\end{array}$ & $\left|\begin{array}{l}0.013 \\
(0.004)\end{array}\right|$ & $\begin{array}{l}0.76 \\
(0.18)\end{array}$ & $\begin{array}{l}12 \\
(0)\end{array}$ & $\begin{array}{l}5 \\
(1)\end{array}$ & $\begin{array}{l}0.0717 \\
\text { (n.s.) }\end{array}$ \\
\hline
\end{tabular}
nonsynonymous site; $d S$ = number of synonymous SNPs per synonymous site. Mixed SNPs are polymorphic in EGs but fixed in PGs, meaning that at least one EG contains a PG-like allele. The genes listed have mixed NS, mixed NS:S, and mixed $d N / d S$ each over two standard deviations above their respective genome-wide medians in the primary dataset. A one-sided binomial test determined if the mixed NS:S ratio was greater than the expected genome-wide median value of 0.5 per gene (uncorrected $P$-values shown; asterisks $\left({ }^{*}\right)$ indicate $P<0.05$ after Bonferroni correction for five tests). Numbers in parentheses are for the replication dataset, with an expected genome-wide median mixed N:S of 0.33 . P-values greater than 0.1 are denoted as not significant (n.s.). Genes 1, 2, 4, and 5 are indicated with arrows on Figure 1C. Gene 3 is on chromosome 2 (Supplementary Fig. 3). 
a Genome-wide phylogeny

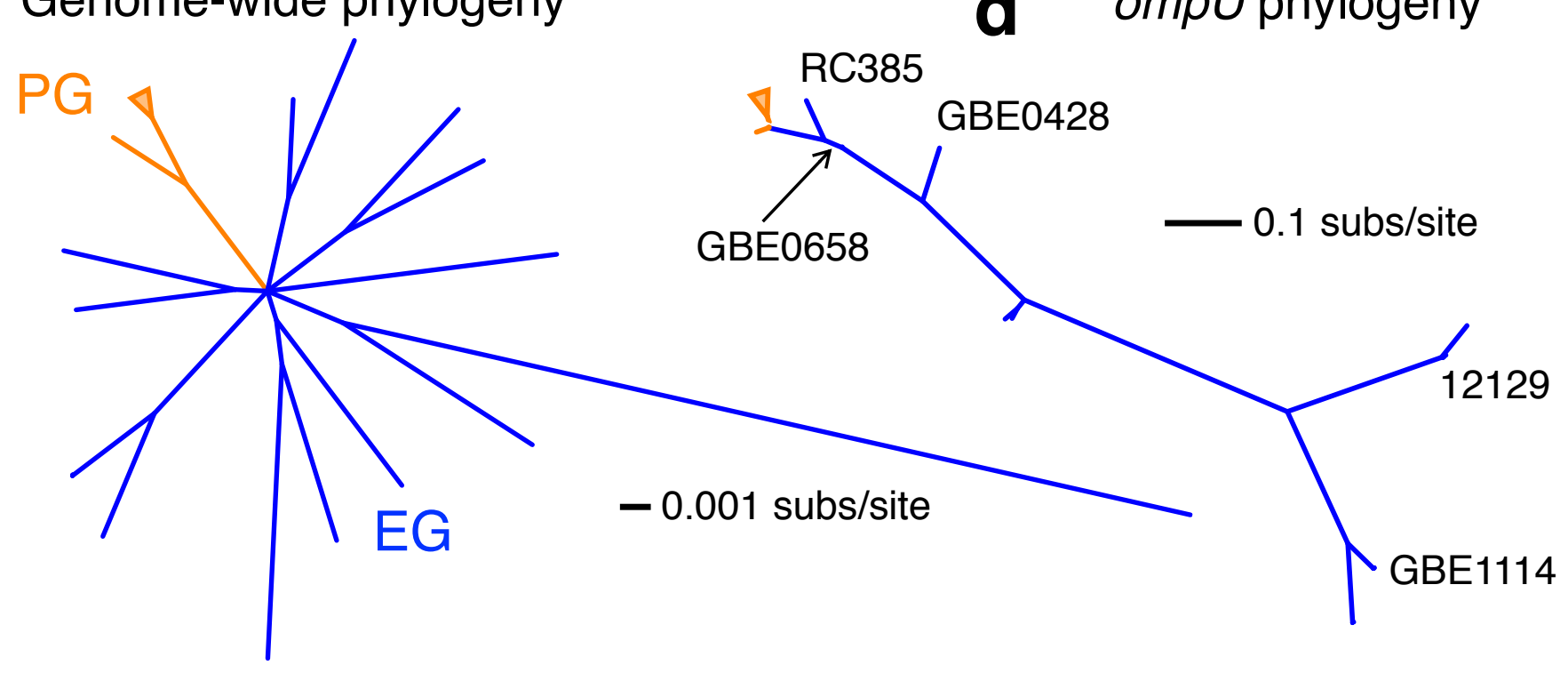

b $\bullet$ FIXED SNPs $\bullet$ total dimorphic SNPS

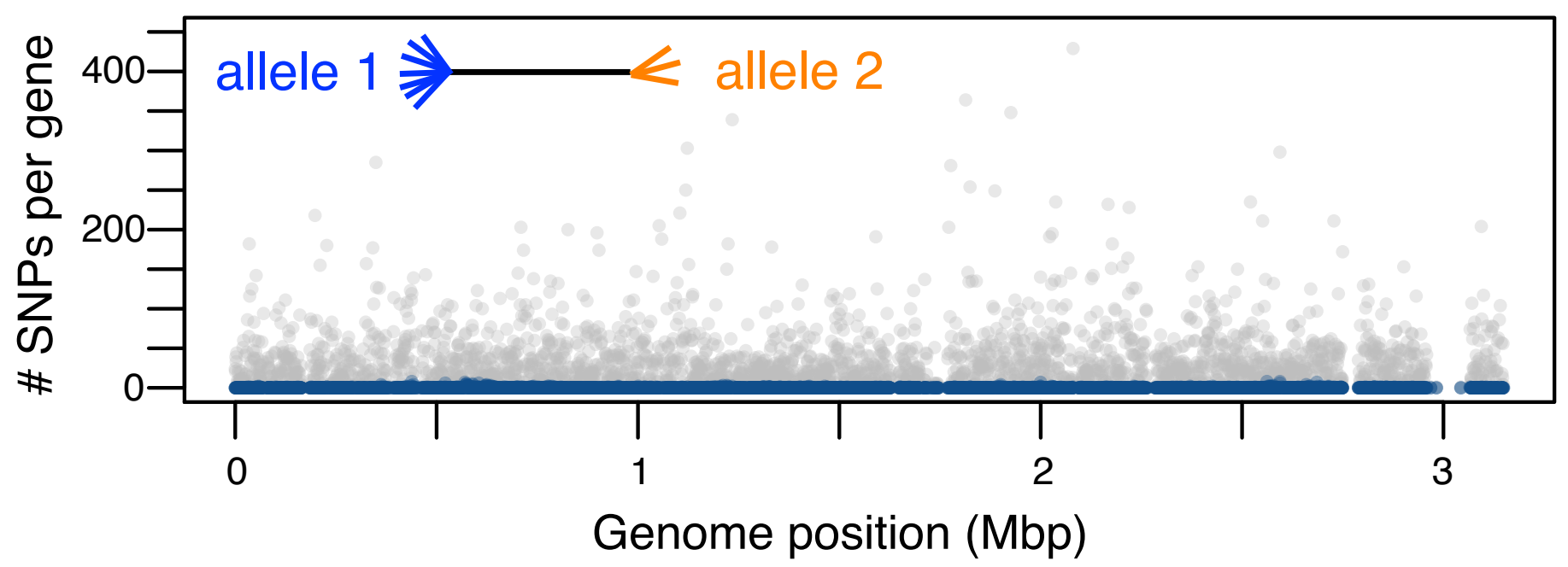

C $\bullet$ MIXED SNPs (nonsynonymous, synonymous) $\bullet$ total dimorphic SNPs

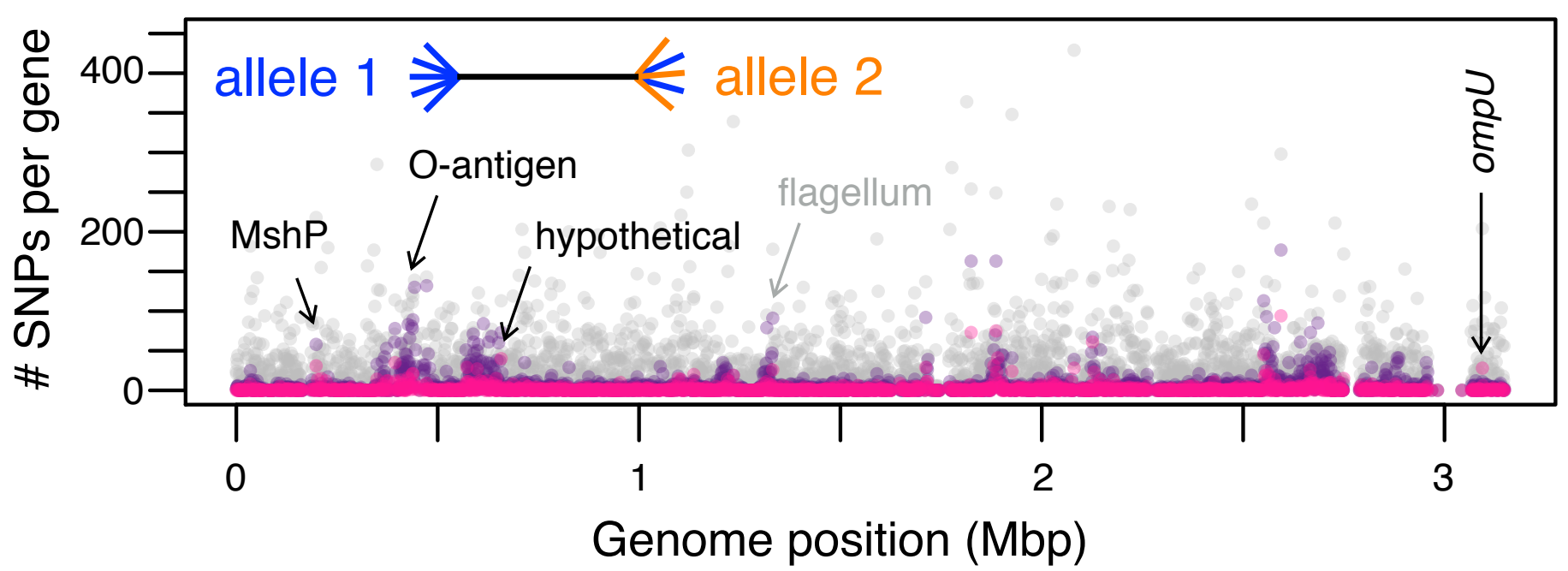


a

37.7 kDa

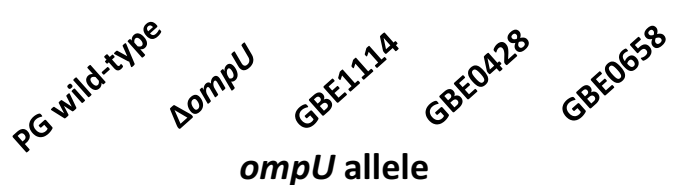

b $\left.\quad{ }^{100}\right] \top$

*** T

bioRxiv preprint doi: https://doi.org/10 1101/063115; this version posted Dece not certified by peer review) is the duthor/funder, who has granted bioRxiva license to display the preprint in perpetuity. It is made availa
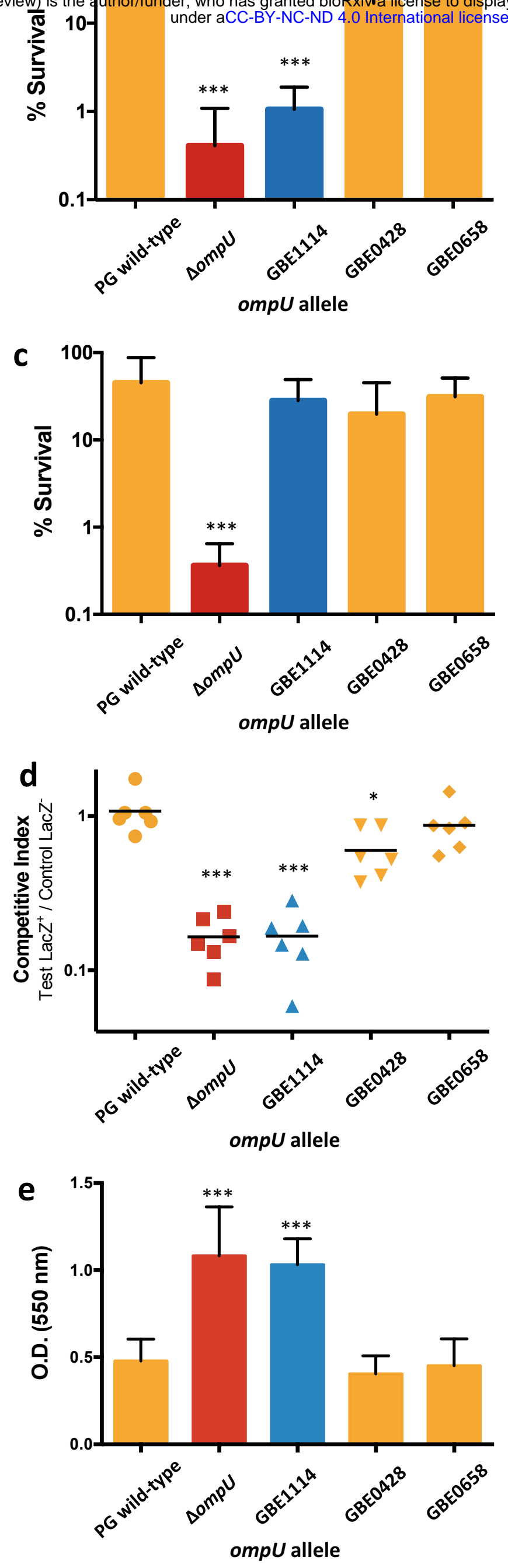
a

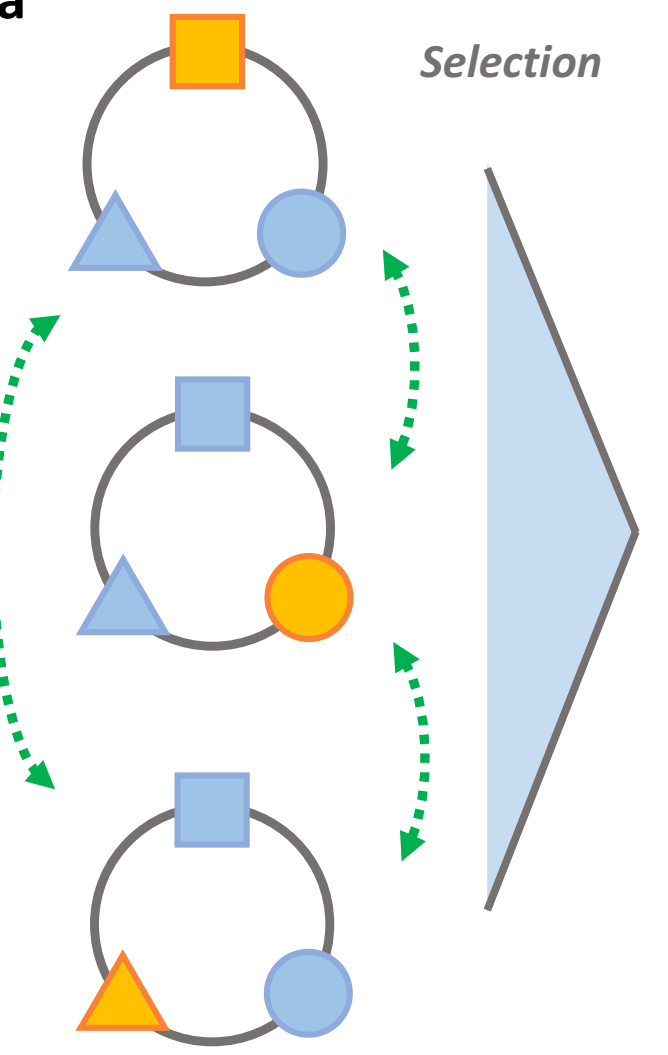

b

Enrichment

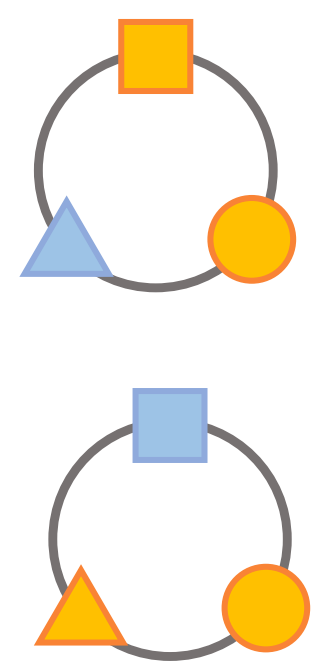

C

Acquisition of

virulence factors

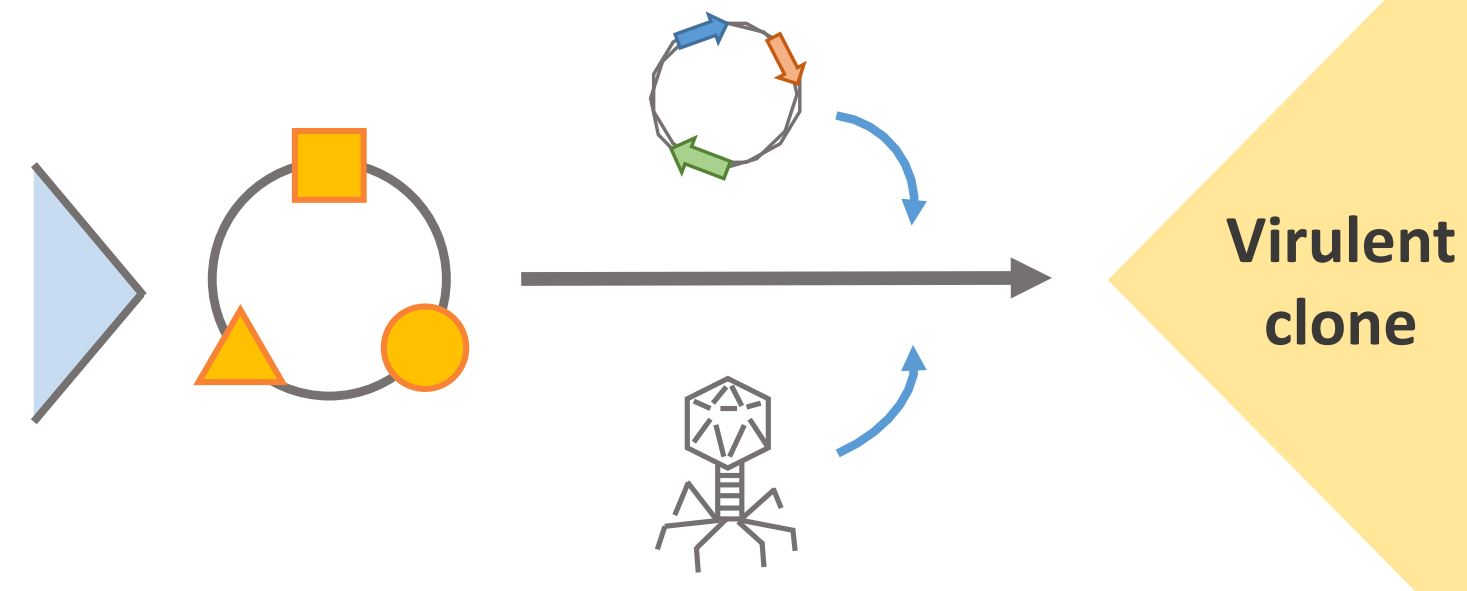

PG-like allele

EG-like allele 